\title{
Extracellular matrix (ECM) activates $\beta$-catenin signaling in uterine fibroids
}

\author{
Yi-An Ko${ }^{1}$, M Fairuz B Jamaluddin ${ }^{1}$, Mariam Adebayo ${ }^{1}$, Preety Bajwa ${ }^{1}$, Rodney J Scott ${ }^{1,5}$, \\ Arunasalam M Dharmarajan², Pravin Nahar ${ }^{3,4}$ and Pradeep S Tanwar ${ }^{1}$ \\ ${ }^{1}$ School of Biomedical Sciences and Pharmacy, University of Newcastle, Callaghan, New South Wales, Australia, \\ ${ }^{2}$ Stem Cell and Cancer Biology Laboratory, School of Biomedical Sciences, Curtin Health Innovation Research \\ Institute, Curtin University, Perth, Western Australia, Australia, ${ }^{3}$ School of Medicine and Public Health, University of \\ Newcastle, Callaghan, New South Wales, Australia, ${ }^{4}$ Department of Maternity and Gynecology, John Hunter \\ Hospital, New Lambton, New South Wales, Australia and ${ }^{5}$ Division of Molecular Medicine, NSW Health Pathology, \\ John Hunter Hospital, Newcastle, New South Wales, Australia
}

Correspondence should be addressed to P S Tanwar; Email: pradeep.tanwar@newcastle.edu.au

\begin{abstract}
Recent studies showed that genetic aberrations in the MED12 gene, probably through the canonical WNT/ $\beta$-catenin pathway, lead to the pathogenesis of uterine fibroids. However, a comprehensive analysis of the WNT pathway in MED12-mutated and MED12-wildtype fibroids has not been performed. The objective of this study was to determine the status of the WNT pathway in human fibroids. We performed Sanger sequencing to define the MED12 mutational status of fibroids and normal myometrium samples. qPCR arrays were carried out to determine the status of the WNT signaling pathway in MED12-mutated and MED12-wild-type fibroids. Liquid chromatography-mass spectrometry (LC-MS), Western blotting and immunohistochemistry were used to monitor the expression of $\beta$-catenin. We showed that $\beta$-catenin expression was increased in fibroids compared to the adjacent myometrium samples. However, $\beta$-catenin expression showed no correlation with MED12 mutation status. Of all the WNT signaling components, WNT inhibitors showed the greatest differences in expression between fibroids and controls. WIF1, a WNT inhibitor, was identified as the most significantly upregulated gene in fibroids. We cultured primary fibroid cells on hydrogels of known stiffness to decipher the influence of biomechanical cues on $\beta$-catenin expression and revealed increased levels of $\beta$-catenin when cells were cultured on a stiffer surface. In conclusion, our data showed that $\beta$-catenin expression in fibroids occurs independently of MED12 mutations.

Biomechanical changes upregulate $\beta$-catenin expression in fibroids, providing an attractive avenue for developing new treatments for this disease.
\end{abstract}

Reproduction (2018) 155 61-71

\section{Introduction}

Uterine leiomyomas, also known as fibroids, are hormone-dependent benign tumors that occur in $60 \%$ of premenopausal women (Okolo 2008). The majority of these women present with symptoms including heavy or prolonged menstrual bleeding, pelvic pain and bowel and bladder dysfunction due to excessive pressure applied by the tumors (Okolo 2008, Bulun 2013). Fibroids, especially at the submucosal location, are also associated with infertility and abortion (Bulun 2013). Surgical methods such as hysterectomy and myomectomy are still considered the main treatment for this disease. Fibroids account for $35 \%$ of all hysterectomies performed in the USA, which represents a very high economic cost on health care (Cramer \& Patel 1990).

Recent advances in gene sequencing approaches have revealed significant heterogeneity in fibroids. The most common driver mutations are found in high mobility group AT-hook 2 (HMGA2), fumarate hydratase $(F H)$, mediator subunit 12 (MED12) and collagen type IV, alpha 5 and 6 (COL4A5-COL4A6) (Mehine et al. 2013). Among the four mutation subtypes, the most prevalent mutations are in the MED12 gene, which contributes to $70 \%$ of the tumors (Mäkinen et al. 2011). MED12 is a part of a large protein complex that mediates gene expression by assisting the communication between DNA binding transcription factors and RNA polymerase (Conaway et al. 2005). Med12 was shown to be essential for early mouse development via the WNT signaling pathway (Rocha et al. 2010) and the mediator complex can bind directly to $\beta$-catenin via the MED12 subunit to assist transcription (Kim et al. 2006). Many different groups have identified hyper-activation of WNT/ $\beta$ catenin signaling in fibroids (Bulun 2013, Zaitseva et al. 2013). We developed a mouse model and demonstrated 
that sustained activation of $\beta$-catenin signaling drives the growth of fibroids that are histopathologically similar to human fibroids and responsive to changes in ovarian hormones (Tanwar et al. 2009). Treatment of human fibroid cells in vitro with WNT/ $\beta$-catenin inhibitors has been shown to suppress their growth and proliferation, suggesting that targeting this pathway might be an effective therapeutic strategy against this disease (Ono et al. 2014). Despite the significance of WNT/ $\beta$ catenin signaling in uterine leiomyogenesis, no genetic alterations affecting the WNT pathway have been identified in fibroids. Additionally, there is currently very little evidence to support that MED12 mutations/ deletions act through WNT/ $\beta$-catenin signaling to drive the process of fibroid development.

In this study, we have performed a comprehensive analysis of the entire WNT pathway in fibroids with, and without, MED12 mutations. We examined at both the mRNA and the protein level to uncover the relationship between MED12, WNT/ $\beta$-catenin signaling and fibroid growth. We have also compared the status of WNT/ $\beta$-catenin signaling in fibroids within the same patient and between different patients to minimize the influence of patient-to-patient variation. Our assessment of the WNT ligands, receptors, activators, inhibitors and targets revealed that $\beta$-catenin mRNA and protein expression was upregulated in fibroids compared to the adjacent normal myometrium. In contrast to a previously published report (de Graaff et al. 2013), we found that the MED12 mutation status of fibroids did not affect $\beta$-catenin expression, and the expression of WNT inhibitors, DKK1 and SFRP1, was upregulated in fibroids with MED12 mutations compared to those with normal MED12. We have also provided evidence that extracellular matrix $(E C M)$, which is one of the major components of fibroids, upregulates $\beta$-catenin.

\section{Materials and methods}

\section{Human tissue collection}

Human fibroid and the adjacent myometrium samples were collected from patients who underwent hysterectomies at the John Hunter Hospital using a protocol approved by the Institutional Human Research Ethics Committee at the University of Newcastle. The participants gave written informed consent to donate their tissues for this study. For patients with multiple fibroids, small $(<2 \mathrm{~cm}$ diameter), medium $(2.1-4 \mathrm{~cm}$ diameter) and large $(>4 \mathrm{~cm}$ diameter) fibroids were collected separately. Fresh samples were transported immediately to the laboratory in phosphate-buffered saline (PBS) on ice. Samples were extensively washed with PBS to remove excess blood prior to any molecular, cellular and histological analysis.

\section{Sanger sequencing}

To determine the status of MED12 genetic aberrations in our tissue samples, we performed Sanger sequencing using a published protocol (Halder et al. 2015). Genomic DNA extraction from fibroids and normal adjacent tissue samples was performed using QIAamp DNA Mini Kit (Qiagen). The DNA fragment was amplified using specific forward and reverse (F: 5'-GCCCTTTCACCTTGTTCCTT-3' and R: $5^{\prime}$-TGTCCCTATAAGTCTTCCCAACC-3') primers that targeted exon 2 of MED12. Following PCR amplification, the products were purified with QIAquick PCR Purification Kit (Qiagen) and quantified with NanoDrop 2000/2000c Spectrophotometer. The purified PCR products were directly sequenced using the BigDye Terminator method according to the manufacturer's instructions (Life Technologies) and the same PCR primers specific to the exon 2 of MED12.

\section{Human WNT signaling pathway arrays}

RNA was extracted from 32 fresh frozen tissue samples from 13 different patients using TRI Reagent solution (Life Technologies) and further purified by RNeasy MinElute Cleanup kit (Qiagen) with additional RNase-free DNase I treatment. The amount of RNA was quantified using a NanoDrop 2000/2000c Spectrophotometer. Five hundred nanograms of purified RNA were then synthesized into cDNA with the RT ${ }^{2}$ First Strand Kit (Qiagen). All steps were followed as per the manufacturer's instructions. Gene expression in relation to the WNT pathway was quantified using Human WNT Signaling Pathway Plus PCR Array (Qiagen) using the 7900HT Fast Real-Time PCR System (Applied Biosystem). The fold regulation change of each gene for all tumor samples was compared to the respective normal adjacent tissue from each individual patient. Two-way ANOVA (paired $t$-test) was utilized here to identify the differential gene expression in MED12 mutation-positive and -negative tumor samples and between the paired adjacent myometrium and fibroid tissue. Gene with $P$ value $<0.05$ is considered significant. A heat-map showing significantly upregulated genes was generated using Gltools analysis software.

\section{Immunohistochemistry (IHC)}

$\mathrm{IHC}$ for $\beta$-catenin was performed using a protocol described previously (Goad et al. 2017). Briefly, tissues were dewaxed and incubated in a series of xylenes, ethanol and MilliQ water, followed by antigen retrieval in sodium citrate buffer $(\mathrm{pH} 6)$ containing $5 \%$ Tween 20 at $95^{\circ} \mathrm{C}$ for $20 \mathrm{~min}$. Slides were cooled and washed with MilliQ water and TBS-T (tween-20) then followed by quenching with $0.3 \%$ hydrogen peroxide in absolute methanol. After few washes, tissue slides were blocked with $10 \%$ goat serum blocking solution $(0.1 \%$ BSA and $0.1 \%$ triton $\mathrm{x}$ in TBS-T) for one hour followed by overnight incubation at $4{ }^{\circ} \mathrm{C}$ with a primary anti- $\beta$-catenin antibody $(1: 100$, Cat no. D10A8, Cell Signaling Technology). Next, tissue samples were incubated with secondary antibody, goat-anti-rabbit $(1: 250$, Jackson Lab) for one hour. Slides were washed and treated with peroxidase-conjugated Streptavidin (1:250, Jackson Lab) and finally exposed to diaminobenzidine (DAB, Sigma) containing $0.3 \%$ hydrogen peroxide. Slides were imaged using the Aperio AT2 slide scanner (Leica Biosystems). The gain and exposure time was set constant across tissue samples. 


\section{Quantitative proteomics using isobaric tags}

Fibroid samples were cut into small pieces and homogenized in ice-cold lysis buffer $0.1 \mathrm{M}$ sodium carbonate, $10 \mathrm{mM}$ sodium ortho-vanadate, protease inhibitor cocktail (Complete Mini, Roche) and 1 tablet of PhosStop (Roche) and homogenized using the beadbug homogenizer (Benchmark Scientific). The concentrated soluble proteins were dissolved in $12 \mathrm{M} \mathrm{Urea/4M}$ Thiourea buffer, reduced with $10 \mathrm{mM}$ of dithiothreitol, alkylated with $20 \mathrm{mM}$ lodoacetamide and digested with $50 \mu \mathrm{g}$ of trypsin (Promega) overnight at $37^{\circ} \mathrm{C}$. After trypsin digestion, peptides were dried by SpeedVac SC100 vacuum centrifugation. Peptides were reconstituted in the dissolution buffer and processed according to the manufacturer's protocol for 4-plex iTRAQ reagent (Sigma). The peptides were labeled with the isobaric tags, incubated at room temperature for $2 \mathrm{~h}$. The labeled peptide mixtures were then pooled and dried by vacuum centrifugation. The mixed peptides were resuspended in the reconstitution buffer, fractionated by strong cation exchange chromatography and loaded onto a hydrophilic interaction liquid chromatography (HILIC) column. The eluted peptides were collected into 20 fractions, desalted, vacuumdried and stored at $-20^{\circ} \mathrm{C}$ until analyzed by mass spectrometry (MS). MS was performed using Q-Exactive Plus Orbitrap mass spectrometer (Thermo Scientific) and intact peptides, ion fragments were detected in the Orbitrap using specific perimeter resolution. Data analysis and protein identification were performed using Proteome Discoverer software package (Thermo Scientific, version2.0.0.802) and UniProt Human reference proteome database, respectively.

\section{Western blot}

Western blot analysis method has been described in detail by us (Bajwa et al. 2016). Briefly, human leiomyoma tissue samples were homogenized in ice-cold radioimmunoprecipitation assay buffer (RIPA) supplemented with protease and phosphatase inhibitors. Equal amounts of proteins were loaded equally onto 10\% Mini-PROTEAN TGX Precast Protein Gels (BioRad). Membranes were incubated with a primary anti- $\beta$-catenin antibody $(1: 1000$, CST) and $\beta$-actin antibody (1:2000, Developmental Studies Hybridoma Bank, IA, USA) overnight at $4{ }^{\circ} \mathrm{C}$ followed by incubation with HRP-conjugated secondary antibodies against mouse and rabbit IgG in room temperature for one hour. Images were captured with Fujifilm LAS-3000 Imager. The band of Western blot was quantified using NIH ImageJ plugin.

\section{Human leiomyoma cell isolation and culture}

Primary cells were isolated using a published protocol (Kim et al. 2015). Tissue samples were washed with PBS and chopped into very fine pieces followed by digestion with $0.2 \mathrm{mg} / \mathrm{mL}$ collagenase $1 \mathrm{~A}, 0.2 \mathrm{mg} / \mathrm{mL}$ collagenase $\mathrm{XI}$ and $2 \mathrm{mg} /$ $\mathrm{mL}$ bovine serum albumin at $37^{\circ} \mathrm{C}$ for $1 \mathrm{~h}$. The mixture was then filtered with $0.2 \mu \mathrm{m}$ sterile Minisart filter and passaged in DMEM media supplemented with $1 \%$ penicillin-streptomycin, $10 \%$ fetal bovine serum (FBS), and $200 \mathrm{~nm}$ L-glutamine. Cells were incubated at $37^{\circ} \mathrm{C}$ with $5 \% \quad \mathrm{CO}_{2}$. Complete media was changed every alternate day and further passaged with
$0.25 \%$ trypsin for five minutes when the cells reached $70 \%$ confluency. Seeding density was 60,000 cells per $10 \mathrm{~cm}^{2}$ with expected $70 \%$ confluency in $72 \mathrm{~h}$.

\section{Hydrogel preparation and imaging}

Polyacrylamide gel of varying stiffness was prepared as per a published protocol (Tse \& Engler 2010). Briefly, acrylamide and bis-acrylamide were mixed with indicated concentration in Table 1, followed by polymerization for $15 \mathrm{~min}$. Gels were then covered with $0.001 \mathrm{~g} / \mathrm{mL}$ of SulfoSANPAH (sulfosuccinimidyl 6-(4'-azido-2'-nitrophenylamino) hexanoate) (Sigma) and exposed directly under UV light for $10 \mathrm{~min}$. After two washes, the gels were incubated overnight with neutralized $0.2 \mathrm{mg} / \mathrm{mL}$ type I collagen (Sigma) at $4^{\circ} \mathrm{C}$. The isolated leiomyoma cells were plated at a density of 20,000 cells per gel and incubated at $37^{\circ} \mathrm{C}$ for three days prior to immunofluorescent staining and analysis. Hydrogels were fixed with $4 \%$ paraformaldehyde followed by two washes and incubation with primary monoclonal anti- $\beta$-catenin $(1: 100$, CST) overnight at $4{ }^{\circ} \mathrm{C}$. Alexa-Fluor 488 secondary antibodies (1:250; Jackson ImmunoResearch Labs) were used for onehour incubation at room temperature. The image was taken using Olympus DP72 microscope. Images were analyzed using ImageJ software (NIH).

\section{Statistical analysis}

GraphPad Prism 6.0 was used in this study for statistical analyses. Data were presented as mean \pm S.E.M. Student's $t$-test was performed for Western blot analysis to compare $\beta$-catenin protein expression in normal myometrium and fibroid tissue. Two-way ANOVA was applied to all the qPCR analysis to compare the gene expression between normal myometrium and fibroid tissue and also between MED12 mutationpositive and -negative tissue. $P$ value $<0.05$ was considered statistically significant.

\section{Results}

\section{Differential expression of WNT pathway members in human uterine fibroids and adjacent normal tissue samples}

The WNT signaling pathway can be divided into three major arms, namely, canonical WNT, WNT/planar cell polarity (PCP) and WNT/Ca+ pathway. Each pathway activates a different set of downstream effectors that are responsible for different actions such as cell proliferation, planar cell polarity and calcium channel activation, respectively. To determine whether WNT

Table 1 Composition of hydrogels of varying stiffness.

\begin{tabular}{lccc}
\hline & $\mathbf{3 ~ k P a}$ & $\mathbf{1 2} \mathbf{~} \mathbf{P a}$ & $\mathbf{4 0 ~} \mathbf{~ P a}$ \\
\hline $10 \mathrm{mM}$ HEPES $(\mu \mathrm{L})$ & 806 & 716 & 560 \\
$40 \%$ Acrylamide $(\mu \mathrm{L})$ & 137.2 & 188.8 & 200 \\
$2 \%$ bis-acrylamide $(\mu \mathrm{L})$ & 44.96 & 80 & 240 \\
APS $(\mu \mathrm{L})$ & 5 & 5 & 5 \\
TEMED $(\mu \mathrm{L})$ & 0.5 & 0.5 & 0.5 \\
\hline
\end{tabular}

Reproduction (2018) 155 61-71 
signaling is altered in uterine fibroids, we collected both fresh fibroids and adjacent normal tissue samples from each patient undergoing hysterectomy $(n=13$; Fig. 1A). Next, we analyzed the expression of 84 genes belonging to the WNT pathway using qPCR arrays to compare the level of their expression between fibroids $(n=19)$ and the adjacent normal tissue samples $(n=12)$. Genes representing different components of the WNT pathway that were significantly differentially expressed between fibroids and normal adjacent tissue samples are presented in the heat-map $(P<0.05$; Fig. 1B). Interestingly, $\beta$-catenin expression was significantly upregulated in fibroids compared to controls $(P=0.004$; Fig. 1C). To further determine the changes in different components of the WNT pathway, we separated all the genes examined in this study into WNT ligands, WNT receptors, WNT inhibitors and WNT targets based on their established roles in previous studies (Fig. 1D). Of all the WNT ligands, WNT2B, WNT5B and WNT9A showed the most significant downregulation in fibroid tissue compared to adjacent normal tissue samples (Fig. 1D). None of the WNT ligands expression was upregulated compared to controls (Fig. 1D). The most significant differences in WNT receptor expression was in Frizzled Class Receptor 6 (FZD6), Low-density Lipoprotein Receptor-Related Protein 5 (LRP5) and Low-density Lipoprotein Receptor-Related Protein 6 (LRP6) and all were downregulated in fibroids (Fig. 1D). Interestingly, the most distinctive changes in expression were found in the genes belonging to the WNT inhibitor category; F-Box and WD Repeat Domain Containing 11 (FBXW11), Secreted Frizzled-Related Protein 4 (SFPR4) and WNT Inhibitory Factor 1 (WIF1) were significantly upregulated in fibroids with a noticeably higher than 20-fold increase for WIF1 compared to adjacent normal tissue samples (Fig. 1D). In contrast, expression of the WNT inhibitors: Adenomatous Polyposis Coli (APC), Dickkopf-Related Protein 1 (DKK1), DKK3, SFPR3/ $F R Z B, P R I C K L E 1$, Ras Homolog Gene Family Member A (RHOA) and SRY-related HMG-Box 17 (SOX17), were significantly decreased in fibroids compared to the adjacent normal myometrium (Fig. 1D). The WNT target, Vang-like protein 2 (VANGL2), was the only gene in this category that was significantly upregulated in fibroids. Conversely, expression of other WNT targets: Disabled homolog 2 (DAB2), JUN, Matrix Metallopeptidase 7 (MMP7), Transcription Factor 7 Like 1 (TCF7L1), WNT1 Inducible Signaling Pathway Protein 1 (WISP1), Cytochrome P450 Family 4 Subfamily V Member 2 (CYP4V2), Heat Shock Protein Family A (Hsp70) Member 12A (HSPA12A), was significantly downregulated in fibroids (Fig. 1D).

To investigate further whether these changes in fibroid gene expression related to any specific WNT pathway, we separated the genes based on the three main arms of the WNT pathways, which are canonical WNT, WNT/planar cell polarity (PCP), WNT/Ca ${ }^{++}$pathway, as well as the negative regulators of the WNT pathway (Fig. 2A). Figure 2B outlines the pathway structure and the molecules involved in each path. For canonical WNT signaling, $\beta$-catenin (CTNNB1), SFRP4 and WIF1 expression were increased, whereas $D K K 1, D K K 3$, SOX17 and WNT2B expression were significantly decreased (Fig. 2A). For the WNT/PCP pathway, VANGL2 expression was highly upregulated and $A P C$, PRICKLE1, RHOA and WNT9A expression levels were significantly reduced. For the WNT/Ca ${ }^{++}$pathway, only the ligands were significantly altered; WNT2B, WNT5B and WNT9A showed a significant decrease in expression in fibroid samples (Fig. 2A). Finally, in the group of negative regulators, SFRP4 and WIF1 were the most highly upregulated genes; whereas $D K K 1, D K K 3$, $S O X 17$ and $F R Z B$ were significantly downregulated in fibroid tissue (Fig. 2A). Among the three WNT pathway categories, most of the significantly altered genes, which were PRICKLE1, VANGL2, WNT9A, DKK1, SFRP4 and WIF1, belong predominantly to the PCP pathway and negative regulator category (Fig. 2A).

\section{Expression of the WNT pathway was downregulated in MED12 gene-mutated uterine fibroids compared to MED12 wild-type fibroids}

To examine whether mutations in the MED12 gene have any effect on WNT signaling, we investigated the expression of WNT pathway components at the RNA level using real-time PCR. Firstly, we determined the MED12 mutation status of nineteen fibroids $(n=19)$ and twelve adjacent normal myometrium $(n=12$ tissue samples) using Sanger sequencing as described by Mäkinen and coworkers (Mäkinen et al. 2011). We noticed that all the adjacent normal myometrium samples had wild-type MED12, whereas some fibroids harbored mutations in MED12 and multiple mutational status was noted in different fibroids taken from one individual. By comparing WNT signaling pathway expression between MED12-mutated and wild-type fibroid samples, we found that CTNNB1 ( $\beta$-catenin) expression did not significantly change with MED12 mutation status ( $P=0.207, n=19$; Fig. $3 \mathrm{~A}$ and $\mathrm{B})$. Comparative analysis of MED12 mutation-positive and MED12 mutation-negative fibroid samples revealed significant differences in the levels of gene expression (Fig. 3C). Of all the genes, FBXW1/BTRC, E1A-associated protein p300 (EP300), FBXW11, IUN, SOX17 and TCF7L1 had significantly decreased expression levels in the MED12-mutation-positive fibroids compared to the MED12 wild type (Fig. 3D). Conversely, expression of SFRP1, WNT2, DKK1 and Lymphoid enhancer-binding factor (LEF1) was increased in those fibroids with MED12 mutations (Fig. 3D). Intriguingly, we did not find any difference in WNT4 expression between MED12-mutated and MED12-wild-type fibroids (Fig. 3A); this finding is in 
A

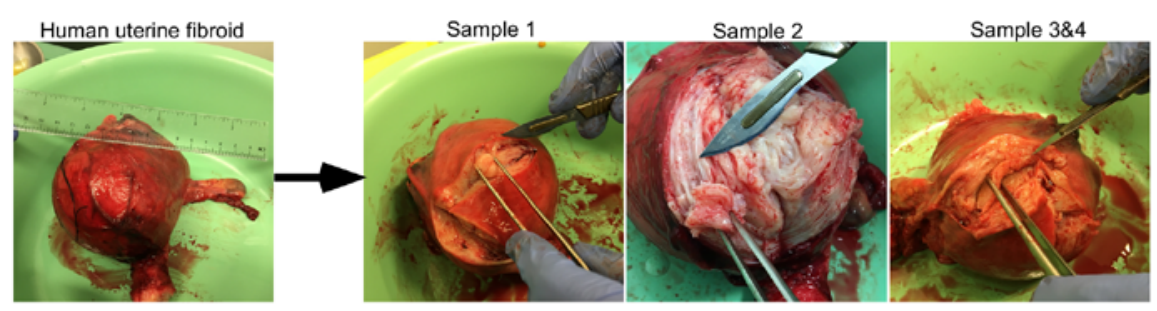

B

B Normal
CTNNB1
DAB2
DKK1
DKK3
FBXW11
FRZB
FZD6
JUN
LRP5
LRP6
MMP7
PRICKLE1
RHOA
SFRP4
SOX17
TCF71
VANGL2
WIF1
WISP1
WNT2B
WNT5B
WNT9A
CYP4V2
HSPA12A

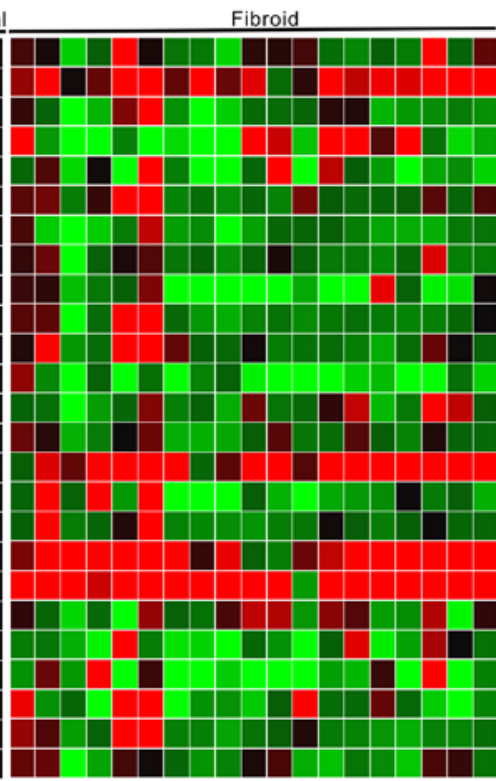

D
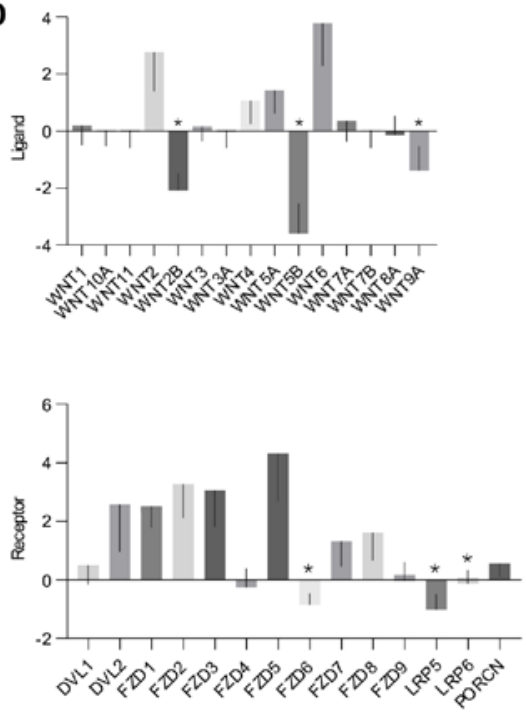

C
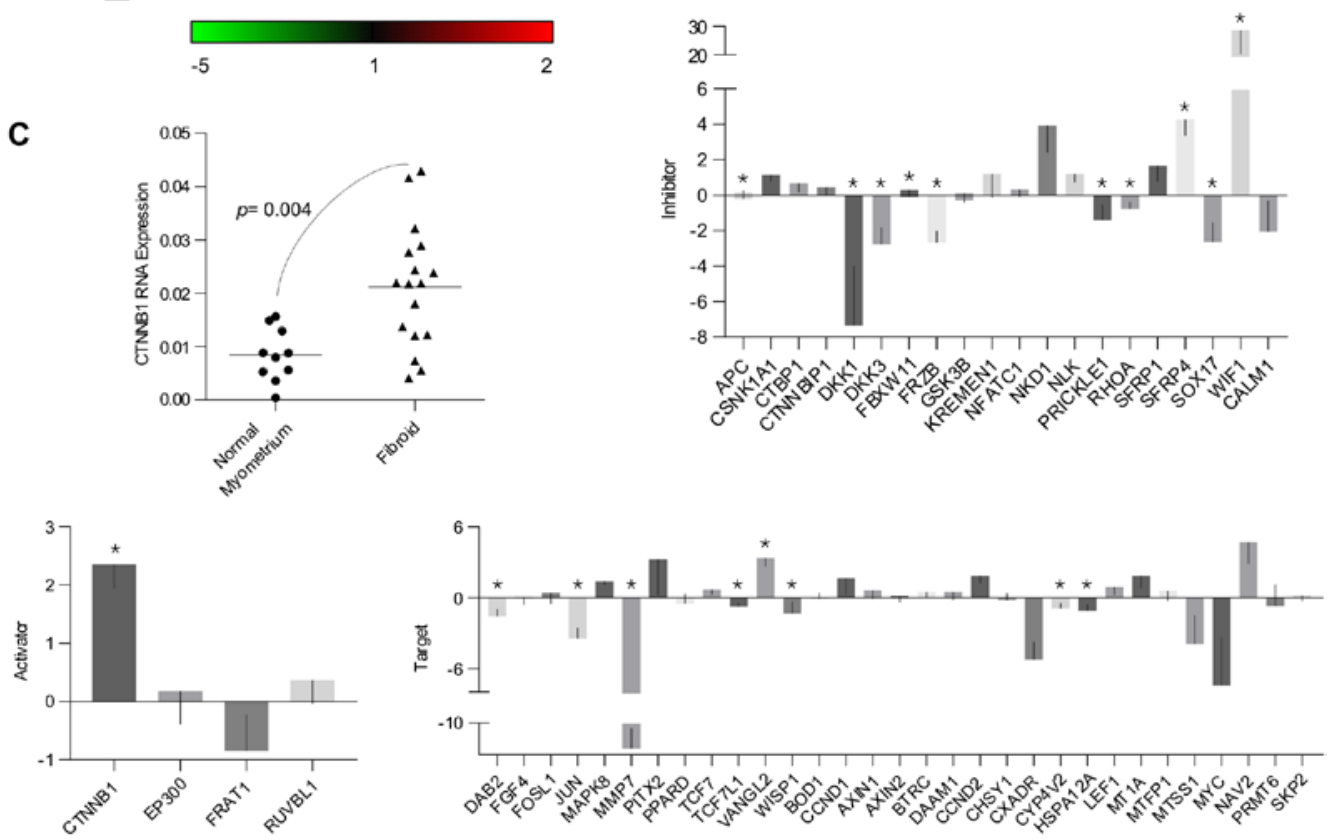

Figure 1 Differential expression of WNT signaling components in fibroids and normal myometrium. (A) Representative pictures of multiple fibroids collected from the same patient. (B) RNA from each tissue sample was extracted and quantified by qPCR. The genes belonging to the WNT pathway that were significantly $(P<0.05)$ different in fibroid from the adjacent normal myometrium were listed in a heat-map. The panel was constructed with 19 fibroid tissue from 13 patients, samples were normalized with its own normal adjacent myometrium. (C) Comparison of $\beta$-catenin expression between normal adjacent myometrium with fibroids $(P<0.05)$. (D) Grouping of different genes into WNT ligands, receptors, activators, inhibitors and targets based on their reported functions. The expression level was normalized with paired myometrium, the relative values were used to generate the bar graph. ${ }^{*} P<0.05$. 

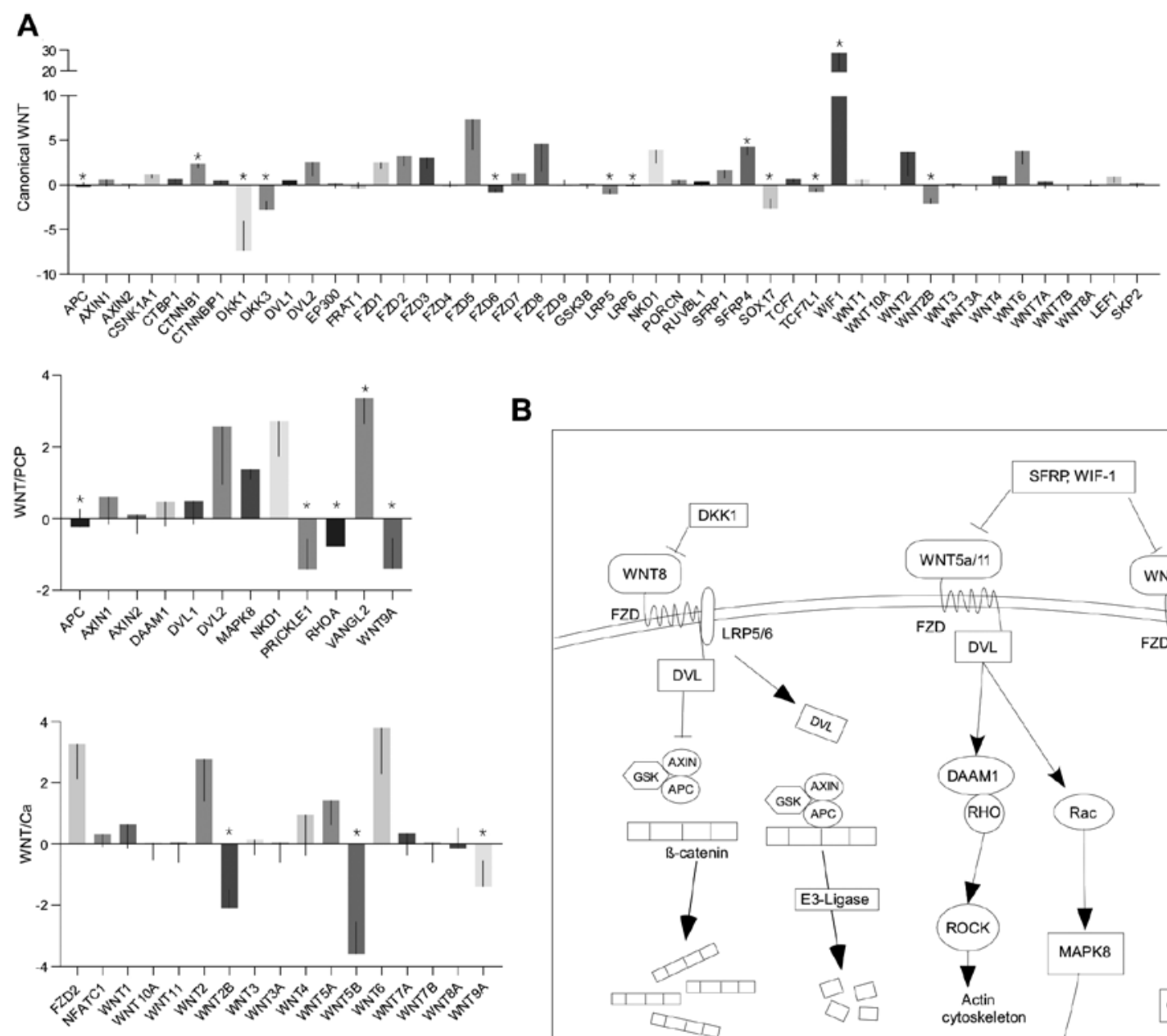

B
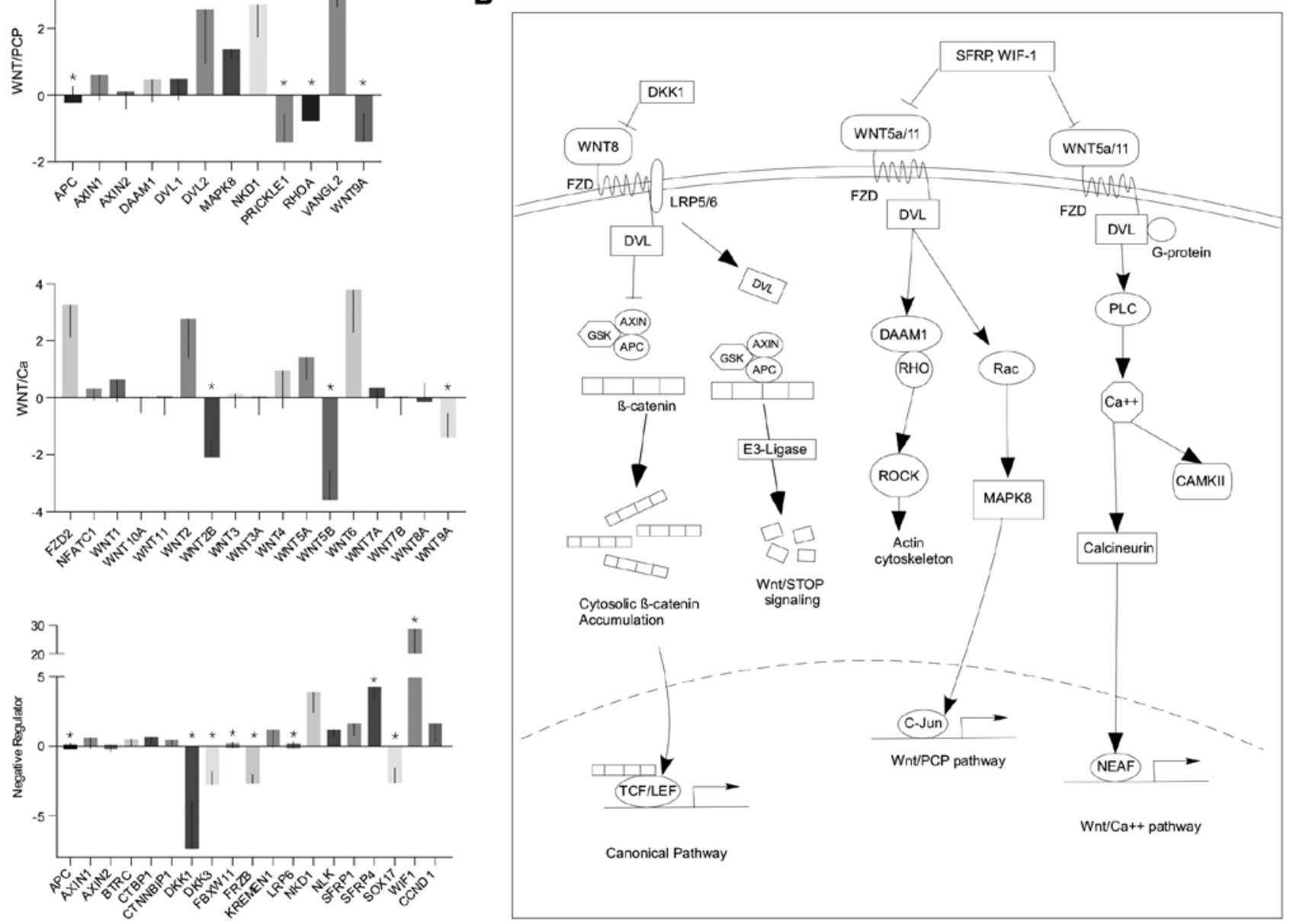

Figure 2 WNT pathway-specific alterations in fibroids and adjacent normal myometrium. (A) Grouping of genes based on the components in canonical WNT pathway, the WNT/PCP pathway, the WNT/Ca ${ }^{++}$pathway, and WNT signaling negative regulators. The expression level was normalized with paired myometrium, the relative values were used to generate the bar graph. ${ }^{*} P<0.05$. (B) Schematic diagram of three main pathways: canonical WNT signaling, WNT/PCP, and WNT/Ca ${ }^{++}$pathways.

contrast to a previous study that reported WNT4 to be highly upregulated in MED12-mutated fibroids (Markowski et al. 2012). In opposition to the currently accepted paradigm that MED12-mutations act through the canonical WNT pathway to alter proliferation of fibroid cells (Markowski et al. 2012, Ono et al. 2013, 2014), we did not find any correlation between MED12 mutation status and $\beta$-catenin expression
(Fig. 3A). Additionally, most canonical WNT pathway components did not show significant differences in expression between MED12 mutation-positive and MED12 mutation-negative samples (Fig. 3). This was consistent with findings by de Graaff and coworkers who suggested the involvement of pathways other than canonical WNT signaling in uterine leiomyogenesis (de Graaff et al. 2013). In summary, these results suggest 


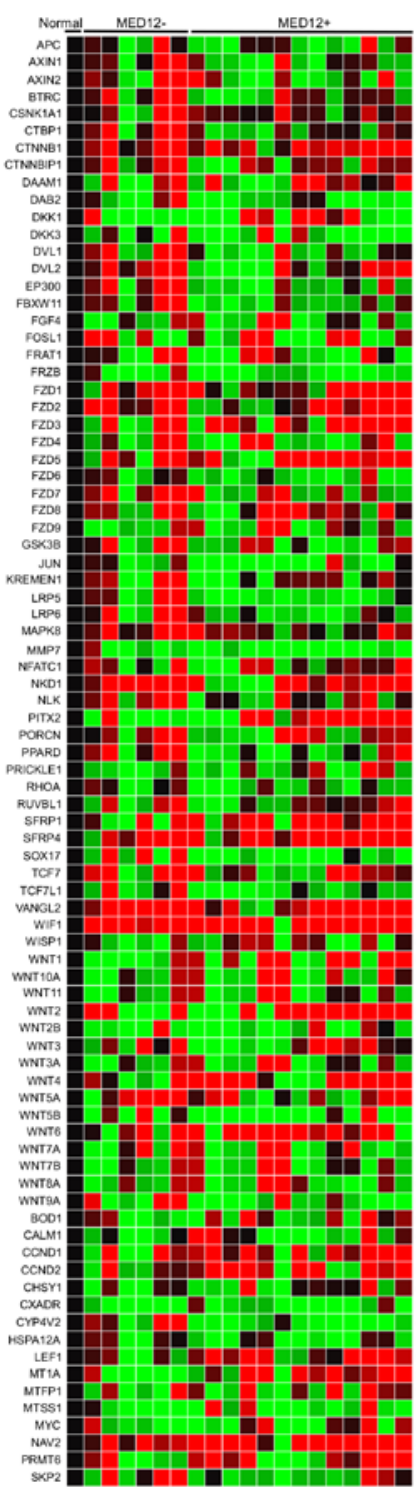

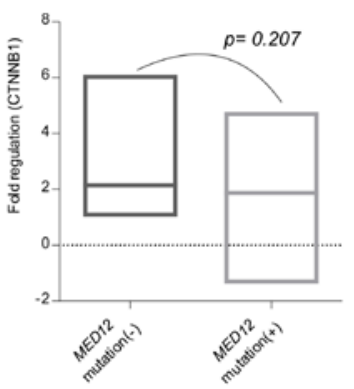

C

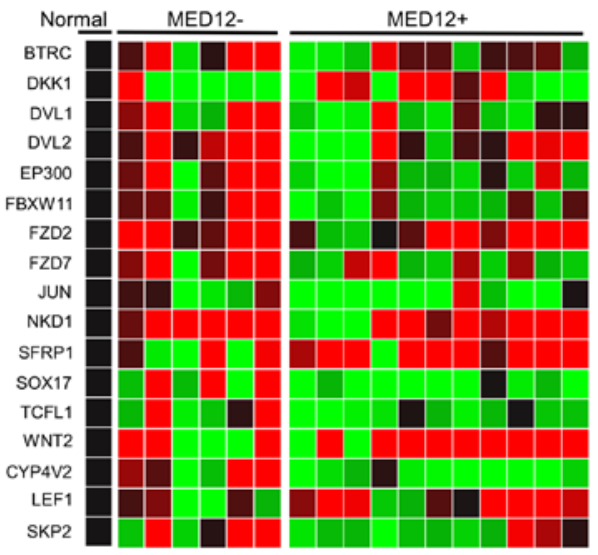

D
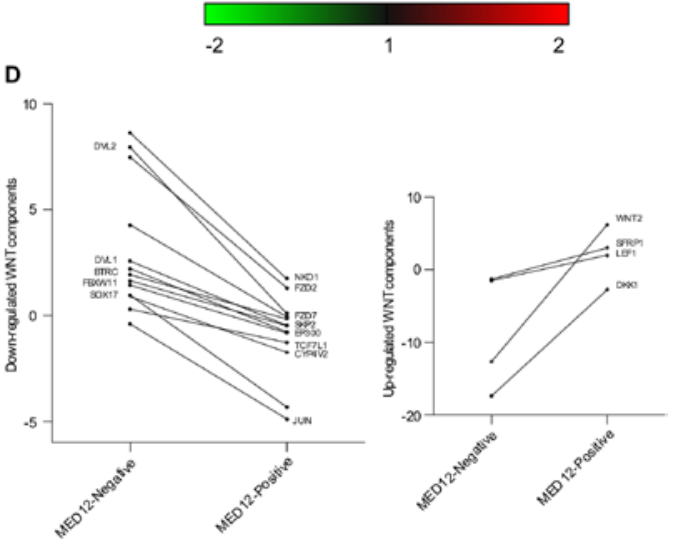

Figure 3 Changes in the expression of the members of the WNT signaling pathway in MED12-mutation-positive (MED12+) and negative (MED12-) fibroids. (A) Analysis of expression of the WNT pathway members in fibroids with and without MED12 mutations. (B) Analysis of $\beta$-catenin expression in fibroids with MED12-mutations and normal MED12 revealed no significant difference between these two groups. (C) Heat-map panel represents the genes of WNT signaling significantly $(P<0.05)$ different between MED12-mutation-positive and MED12-wildtype fibroids. All values were normalized with paired normal myometrium within each patient. (D) Graphs of normalized expression levels for the significantly altered genes of fibroids with and without MED12-mutations.

that genetic alterations in the MED12 gene are unlikely to affect the WNT signaling pathway.

\section{Proteomic analysis of uterine fibroids confirmed that $\beta$-catenin protein expression was increased in uterine fibroids compared to adjacent normal myometrium}

To validate whether $\beta$-catenin expression was increased in fibroids, we used the most advanced method by comparing the proteomic status between adjacent normal myometrium and fibroids from three patients using mass spectrometry. From each patient, we analyzed at least three fibroids and one adjacent normal tissue sample (Fig. 1). In agreement with our RNA data (Figs 1 and 2), we observed upregulation of $\beta$-catenin protein expression in fibroids compared to controls (Fig. 4A). In accordance with our array results (Fig. 3), $\beta$-catenin protein expression pattern was not correlated with MED12-mutation status. The proteomic data were confirmed using Western blot analysis (Fig. 4B). Quantification of protein bands revealed significant differences in $\beta$-catenin protein expression $(P=0.0175)$ 
A
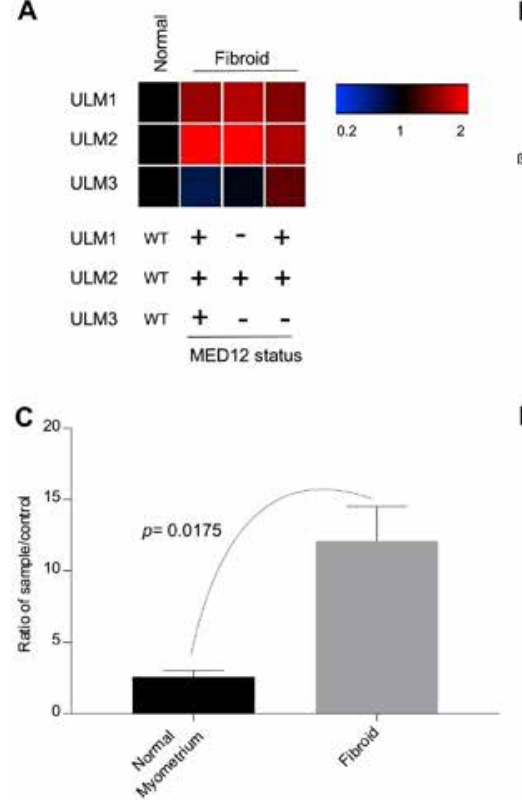

B

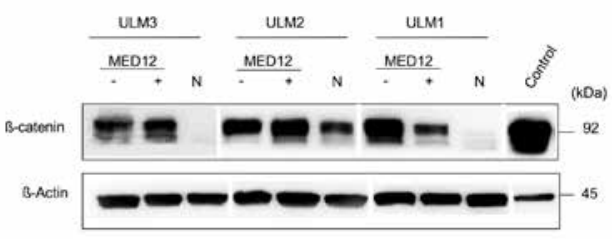

D

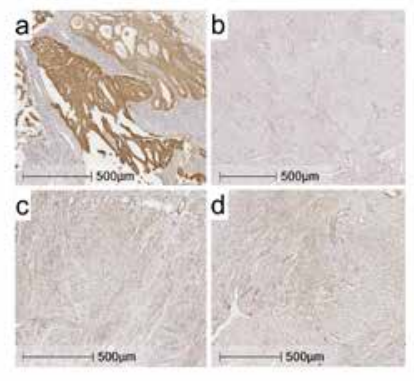

Figure $4 \beta$-Catenin protein expression in fibroids was increased compared to the adjacent normal myometrium independent of the MED12-mutation status. (A) Mass spectrometry analysis of $\beta$-catenin in fibroids. Three fibroids and adjacent normal myometrial tissue samples were analyzed from each patient. (B) Equal amounts of tissue lysates from three patients were analyzed for $\beta$-catenin expression using Western blot. $\beta$-Actin was used as loading control. HEC $1 \alpha$ cell line protein was used as a positive control for $\beta$-catenin expression. (C) Protein quantification of Western blot using $\mathrm{NIH}$ Image J. The intensity of $\beta$-catenin level for each patient was normalized with the corresponding control $\beta$-actin. (D) Immunohistochemistry of $\beta$-catenin. (a) Endometrium tissue used as a positive control. (b) Adjacent normal myometrium tissue, (c and d) uterine leiomyomas. between myometrium and fibroids (Fig. 4C). The $\beta$-catenin protein expression was further validated with immunohistochemistry and showed relatively higher expression in fibroids compared to adjacent myometrium (Fig. 4Db, c and d). Human endometrium was used as a positive control for $\beta$-catenin staining (Fig. 4Da). Altogether, our protein analysis further supports our qPCR profiling whereby $\beta$-catenin appears to be upregulated at both RNA and protein levels in fibroids compared to adjacent normal myometrium.

\section{Increase in ECM stiffness triggers the upregulation of $\beta$-catenin in fibroid cells}

Our extensive analysis of the WNT pathway, both at the RNA and protein level, showed that $\beta$-catenin is overexpressed in fibroids compared to adjacent normal myometrium (Figs 1, 2, 3 and 4). However, we found no evidence for the upregulation of upstream positive regulators (WNT ligands) of $\beta$-catenin and instead, many negative regulators of WNT signaling were expressed at higher levels in fibroids compared to the adjacent normal myometrium (Figs 1, 2, 3 and 4). These findings suggest that $\beta$-catenin overexpression in fibroids does not occur through canonical WNT signaling and possibly involves alternative signaling mechanisms. To determine if an increase in the ECM stiffness leads to augmented $\beta$-catenin expression in uterine leiomyomas, we isolated primary fibroid cells and cultured these cells on hydrogels of known stiffness $(n=3)$. Similar to the primary tumor sample, these cultured cells had a mutation in MED12 (c.130G >A, p.G44S). We performed immunofluorescent staining to examine the level of $\beta$-catenin expression. The results revealed a significant increase in $\beta$-catenin expression with increasing stiffness (Fig. 5A and B). In conclusion, these data highlight that changes in mechanical stiffness lead to increased expression of $\beta$-catenin in fibroids.

\section{Discussion}

MED12 is a subunit of the mediator complex, which mediates gene expression by communicating between transcription factors and RNA polymerase (Allen \& Taatjes 2015). Mutations in MED12 are the most common genetic alterations observed in human uterine leiomyomas, present in approximately $70 \%$ of cases (Mäkinen et al. 2011). Previous studies have shown Med12 conditional knockout mice that express a common variant (c.131G>A), present with an earlier onset of uterine leiomyomas (Mittal et al. 2015), suggesting that this Med12 mutation is sufficient to drive leiomyogenesis. Med12 is essential for early mouse development because the loss of this gene, accompanied with abnormal Wnt/PCP and canonical Wnt signaling, results in embryonic defects in heart formation, axis elongation and somitogenesis (Rocha et al. 2010). In 2006, Kim and coworkers showed by performing immunoprecipitation that the mediator complex can bind directly to $\beta$-catenin through the MED12 subunit (Kim et al. 2006). The interface between $\beta$-catenin and the MED12 subunit was found between codon 1651 and 2086 of MED12. Whether the common MED12 genetic aberrations in codon 44 affects the binding between $\beta$-catenin and the mediator complex remains unknown because the crystal structure of the MED12 protein is yet to be characterized.

Many studies in the fibroid field have suspected the involvement of aberrant WNT signaling in the development of uterine fibroids (Bulun 2013). 
A

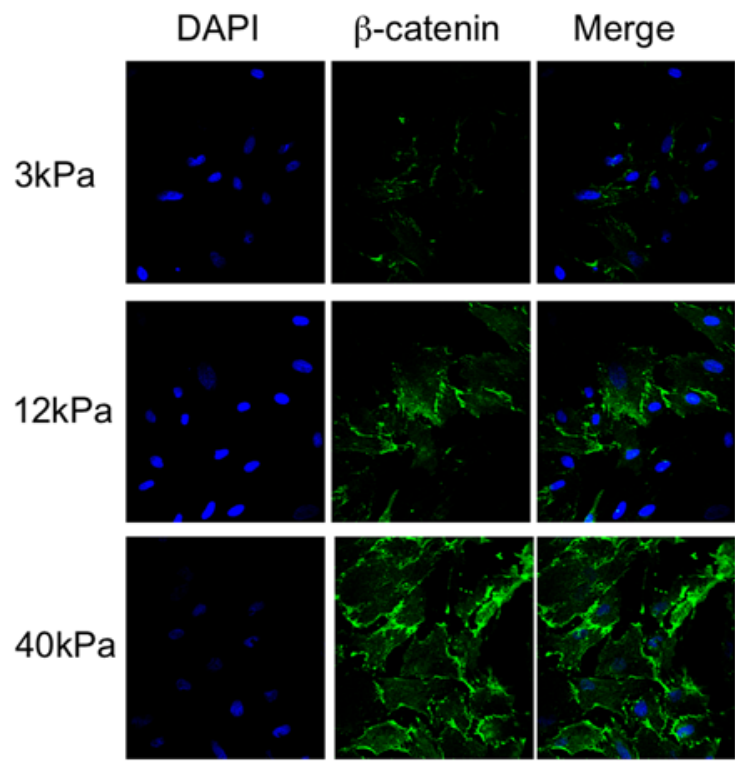

B

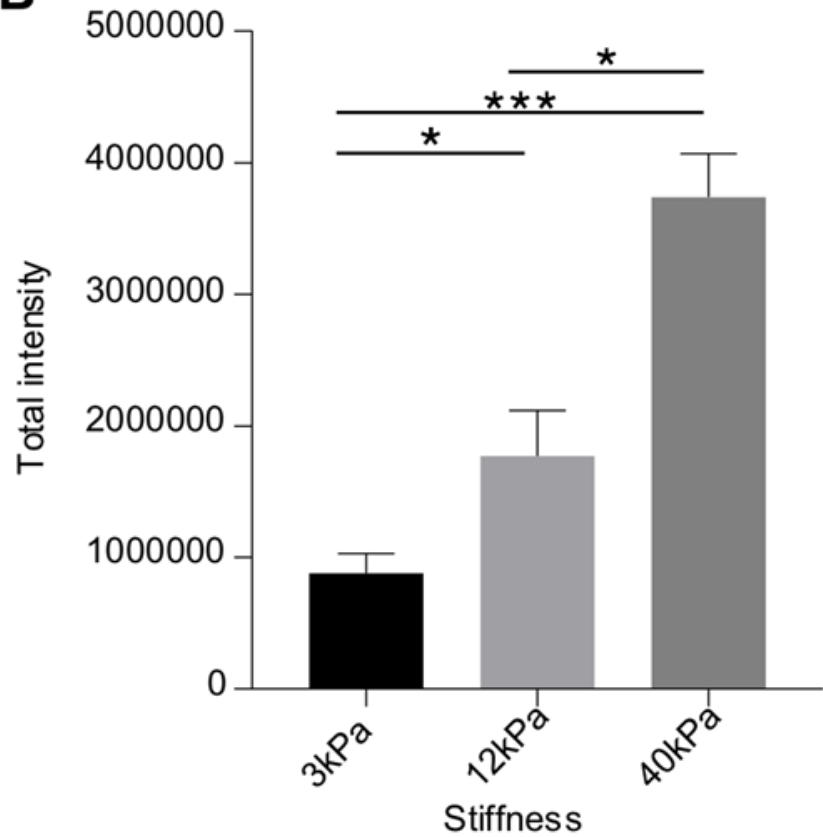

Figure 5 ECM stiffness regulates $\beta$-catenin expression. Primary fibroids cells were extracted and seeded onto hydrogels with three different stiffness, 3, 12, and $40 \mathrm{kPa}$. (A) Representative immunofluorescence images of primary fibroid cells with increase stiffness. (B) Intensity quantification of immunofluorescence of $\beta$-catenin in three different hydrogel stiffness.

Indeed, over-activation of $\beta$-catenin in mouse uterine mesenchyme leads to the development of mesenchymal tumors that are similar to human uterine leiomyomas and tumors of Med12 mouse models (Tanwar et al. 2009). However, no activating mutations in $\beta$-catenin (CTNNB1) or any other member of the WNT pathway have been identified in human fibroid patients despite reports by many groups of the overexpression of $\beta$-catenin protein in uterine fibroids (Zaitseva et al. 2006, 2013). These findings have led to the hypothesis, that, somehow, the common MED12 mutations alter WNT signaling, which in turn change the expression of $\beta$-catenin and results in leiomyoma pathogenesis (Markowski et al. 2012). In the present study, we examined the expression of different components of the WNT pathway in fibroids with, and without, MED12 mutations. To minimize the influence of patient-to-patient variations, we also analyzed multiple fibroids from the same patient. Despite our extensive search using different techniques, we did not find any significant differences in the expression of $\beta$-catenin between fibroids with, or without, MED12 mutations. Oddly, we found increased expression of DKK1 and SFRP1, known inhibitors of WNT signaling, in MED12-mutated fibroids compared to MED12-wildtype fibroids suggesting that WNT signaling might be downregulated in fibroids with MED12 mutations. Interestingly, increased expression of SFRP1 in MED12mutated fibroids was also recently reported in another study (Mehine et al. 2016).

ECM is a major component of uterine fibroids and excessive deposition of ECM proteins is responsible for the growth of fibroids and bulk type symptoms (Stewart et al. 2016). It is well established that changes in mechanical properties of tissues play a significant role in the development of organ systems, stem cell functions and pathogenesis of diseases. Du and coworkers using bone marrow mesenchymal stem cells and primary chondrocytes, presented evidence showing a concomitant increase in $\beta$-catenin levels with increasing ECM stiffness via activation of the integrin-FAK pathway, which may trigger further feedback loop in WNT signaling (Du et al. 2016). Uterine leiomyomas are very stiff tumors and are known to have hyperactive integrinFAK signaling. We examined the effect of ECM stiffness on fibroid cells by culturing cells on hydrogels of known stiffness and found that $\beta$-catenin expression upregulates with increase in stiffness. These results provide a novel insight into how changes in the mechanical properties of fibroids cause changes in the expression of biochemical signals such as $\beta$-catenin.

Our comparative analysis of MED12 mutationpositive and -negative fibroids revealed that $\beta$-catenin expression is not affected by the alterations in MED12. Our examination using primary fibroid cells provided evidence that ECM stiffness affects $\beta$-catenin expression. A recent study by Bloch and coworkers showed that MED12-mutation-positive cells progressively disappear during in vitro culture (Bloch et al. 2017). We performed Sanger sequencing on primary fibroid cells after three passages and still, able to detect the same MED12 mutation that was present in the primary tumor. The differences in our results and results from study by Bloch and coworkers are probably because of different culture conditions (Bloch et al. 2017). Bloch and coworkers 
have used RPMI-1640 supplemented with $20 \%$ fetal calf serum, $100 \mathrm{IU} / \mathrm{mL}$ penicillin and $100 \mu \mathrm{g} / \mathrm{mL}$ streptomycin for culturing fibroid cells, whereas we are using DMEM media supplemented with $1 \%$ penicillin-streptomycin, $10 \%$ fetal bovine serum and $200 \mathrm{~nm}$ L-glutamine. The lack of glutamine could have a profound effect on cell growth and survival.

One of the most surprising findings from our study was the observation that of all the WNT pathway members examined, WNT inhibitors showed the most significant difference in expression between fibroids and adjacent normal myometrium. For example, WIF1 expression was on average 20-fold higher compared to normal adjacent myometrium. Similarly, expression of APC, DKK1, DKK3, FBXW11, PRICKLE1, RHOA, SFRP4 and SOX17 was significantly altered in fibroids compared to controls. Consistent with our findings, a previous study reported overexpression of SFRP1 at both RNA and protein levels in uterine leiomyomas compared to the paired adjacent myometrium; SFRP1 appears to exert an anti-apoptotic effect on the tumors (Fukuhara et al. 2002). Similarly, significantly increased levels of the WNT antagonists, WIF1 and SFRP1 were observed in fibroids with alterations in the HMGA2 or MED12 genes (Mäkinen et al. 2014). Collectively, these findings suggest that WNT signaling involvement in uterine leiomyogenesis is complex, and further studies are required to decipher the individual contributions of various components of the WNT pathway to the pathogenesis of this disease.

In summary, we have confirmed increased $\beta$-catenin expression in uterine leiomyomas compared to paired adjacent normal myometrium at both the posttranscriptional and translational level. We also proposed that ECM stiffness is the key to trigger upregulation of $\beta$-catenin through the integrin-FAK pathway.

\section{Declaration of interest}

The authors declare that there is no conflict of interest that could be perceived as prejudicing the impartiality of the research reported.

\section{Funding}

This research did not receive any specific grant from any funding agency in the public, commercial or not-for-profit sector.

\section{Author contribution statement}

$\mathrm{P} \mathrm{B}$ and $\mathrm{P} \mathrm{N}$ : collected samples and clinical information. M F J and R J S: performed proteomic and mutation analysis. Y A Ko: performed qPCR arrays. Y A Ko and M A: cell culture work and analysis. Y A Ko and A M D: analysis of array data. Y A Ko and P S T: wrote the paper. P S T: designed the study, analyzed the data and provided financial support and final approval of the manuscript. All authors approved and commented on the manuscript.

\section{Acknowledgments}

Work in the Tanwar lab was in part supported by funding from the National Health and Medical Research Council, the Australian Research Council, and the Cancer Institute NSW. P B is a recipient of the University of Newcastle Postgraduate Research Fellowship.

\section{References}

Allen BL \& Taatjes DJ 2015 The Mediator complex: a central integrator of transcription. Nature Reviews Molecular Cell Biology 16 155-166. (https://doi.org/10.1038/nrm3951)

Bajwa P, Nagendra PB, Nielsen S, Sahoo SS, Bielanowicz A, Lombard JM, Wilkinson JE, Miller RA \& Tanwar PS 2016 Age related increase in mTOR activity contributes to the pathological changes in ovarian surface epithelium. Oncotarget 7 19214-19227. (https://doi.org/10.18632/ oncotarget.8468)

Bloch J, Holzmann C, Koczan D, Helmke BM \& Bullerdiek J 2017 Factors affecting the loss of MED12-mutated leiomyoma cells during in vitro growth. Oncotarget 8 34762-34772. (https://doi.org/10.18632/oncotarget.16711)

Bulun SE 2013 Uterine fibroids. New England Journal of Medicine 369 1344-1355. (https://doi.org/10.1056/NEJMra1209993)

Conaway RC, Sato S, Tomomori-Sato C, Yao T \& Conaway JW 2005 The mammalian Mediator complex and its role in transcriptional regulation. Trends in Biochemical Sciences 30 250-255. (https://doi.org/10.1016/j. tibs.2005.03.002)

Cramer SF \& Patel A 1990 The frequency of uterine leiomyomas. American Journal of Clinical Pathology 94 435-438. (https://doi.org/10.1093/ ajcp/94.4.435)

de Graaff MA, Cleton-Jansen A-M, Szuhai K \& Bovée JV 2013 Mediator complex subunit 12 exon 2 mutation analysis in different subtypes of smooth muscle tumors confirms genetic heterogeneity. Human pathology 44 1597-1604. (https://doi.org/10.1016/j.humpath.2013.01.006)

Du J, Zu Y, Li J, Du S, Xu Y, Zhang L, Jiang L, Wang Z, Chien S \& Yang C 2016 Extracellular matrix stiffness dictates Wnt expression through integrin pathway. Scientific Reports 6 20395. (https://doi.org/10.1038/ srep20395)

Fukuhara K, Kariya M, Kita M, Shime H, Kanamori T, Kosaka C, Orii A, Fujita J \& Fujii S 2002 Secreted frizzled related protein 1 is overexpressed in uterine leiomyomas, associated with a high estrogenic environment and unrelated to proliferative activity. Journal of Clinical Endocrinology and Metabolism 87 1729-1736. (https://doi.org/10.1210/ jcem.87.4.8375)

Goad J, Ko Y-A, Kumar M, Syed SM \& Tanwar PS 2017 Differential Wnt signaling activity limits epithelial gland development to the antimesometrial side of the mouse uterus. Developmental Biology 423 138-151. (https://doi.org/10.1016/j.ydbio.2017.01.015)

Halder SK, Laknaur A, Miller J, Layman LC, Diamond M \& Al-Hendy A 2015 Novel MED12 gene somatic mutations in women from the Southern United States with symptomatic uterine fibroids. Molecular Genetics and Genomics 290 505-511. (https://doi.org/10.1007/s00438014-0938-x)

Kim S, Xu X, Hecht A \& Boyer TG 2006 Mediator is a transducer of Wnt/ $\beta$ catenin signaling. Journal of Biological Chemistry 281 14066-14075. (https://doi.org/10.1074/jbc.M602696200)

Kim SH, Macintyre DA, Da Silva MF, Blanks AM, Lee YS, Thornton S, Bennett PR \& Terzidou V 2015 Oxytocin activates NF-кB-mediated inflammatory pathways in human gestational tissues. Molecular and Cellular Endocrinology 403 64-77. (https://doi.org/10.1016/j.mce.2014.11.008)

Mäkinen N, Mehine M, Tolvanen J, Kaasinen E, Li Y, Lehtonen HJ, Gentile M, Yan J, Enge M \& Taipale M 2011 MED12, the mediator complex subunit 12 gene, is mutated at high frequency in uterine leiomyomas. Science 334 252-255. (https://doi.org/10.1126/science.1208930) 
Mäkinen N, Heinonen H, Sjöberg J, Taipale J, Vahteristo P \& Aaltonen L 2014 Mutation analysis of components of the Mediator kinase module in MED12 mutation-negative uterine leiomyomas. British Journal of Cancer 110 2246-2249. (https://doi.org/10.1038/bjc.2014.138)

Markowski DN, Bartnitzke S, Löning T, Drieschner N, Helmke BM \& Bullerdiek J 2012 MED12 mutations in uterine fibroids-their relationship to cytogenetic subgroups. International Journal of Cancer 131 1528-1536. (https://doi.org/10.1002/ijc.27424)

Mehine M, Kaasinen E, Mäkinen N, Katainen R, Kämpjärvi K, Pitkänen E, Heinonen H-R, Bützow R, Kilpivaara O \& Kuosmanen A 2013 Characterization of uterine leiomyomas by whole-genome sequencing. New England Journal of Medicine 369 43-53. (https://doi.org/10.1056/ NEJMoa1302736)

Mehine $M$, Kaasinen E, Heinonen H-R, Mäkinen N, Kämpjärvi K Sarvilinna N, Aavikko M, Vähärautio A, Pasanen A \& Bützow R 2016 Integrated data analysis reveals uterine leiomyoma subtypes with distinct driver pathways and biomarkers. PNAS 113 1315-1320. (https://doi. org/10.1073/pnas.1518752113)

Mittal P, Shin Y-h, Yatsenko SA, Castro CA, Surti U \& Rajkovic A 2015 Med12 gain-of-function mutation causes leiomyomas and genomic instability. Journal of Clinical Investigation 125 3280-3284. (https://doi. org/10.1172/JCl81534)

Okolo S 2008 Incidence, aetiology and epidemiology of uterine fibroids. Best Practice and Research Clinical Obstetrics and Gynaecology 22 571-588. (https://doi.org/10.1016/j.bpobgyn.2008.04.002)

Ono M, Yin P, Navarro A, Moravek MB, Coon JS, Druschitz SA, Serna VA, Qiang W, Brooks DC \& Malpani SS 2013 Paracrine activation of WNT/ $\beta$ catenin pathway in uterine leiomyoma stem cells promotes tumor growth. PNAS 110 17053-17058. (https://doi.org/10.1073/pnas.1313650110)

Ono M, Yin P, Navarro A, Moravek MB, Druschitz SA, Gottardi CJ \& Bulun SE 2014 Inhibition of canonical WNT signaling attenuates human leiomyoma cell growth. Fertility and Sterility 101 1441.e1441-1449. e1441. (https://doi.org/10.1016/j.fertnstert.2014.01.017)
Rocha PP, Scholze M, Bleiß W \& Schrewe H 2010 Med12 is essential for early mouse development and for canonical Wnt and Wnt/PCP signaling. Development 137 2723-2731. (https://doi.org/10.1242/dev.053660)

Stewart EA, Laughlin-Tommaso SK, Catherino WH, Lalitkumar S, Gupta D \& Vollenhoven B 2016 Uterine fibroids. Nature Reviews Disease Primers 2 16043. (https://doi.org/10.1038/nrdp.2016.43)

Tanwar PS, Lee H-J, Zhang L, Zukerberg LR, Taketo MM, Rueda BR \& Teixeira JM 2009 Constitutive activation of beta-catenin in uterine stroma and smooth muscle leads to the development of mesenchymal tumors in mice 1. Biology of Reproduction 81 545-552. (https://doi. org/10.1095/biolreprod.108.075648)

Tse JR \& Engler AJ 2010 Preparation of hydrogel substrates with tunable mechanical properties. Current Protocols in Cell Biology 10.16.1110.16.16. (https://doi.org/10.1002/0471143030.cb1016s47)

Zaitseva M, Vollenhoven BJ \& Rogers PA 2006 In vitro culture significantly alters gene expression profiles and reduces differences between myometrial and fibroid smooth muscle cells. Molecular Human Reproduction 12 187-207. (https://doi.org/10.1093/molehr/gal018)

Zaitseva M, Holdsworth-Carson SJ, Waldrip L, Nevzorova J, Martelotto L, Vollenhoven BJ \& Rogers PA 2013 Aberrant expression and regulation of NR2F2 and CTNNB1 in uterine fibroids. Reproduction 146 91-102. (https://doi.org/10.1530/REP-13-0087)

Received 5 June 2017

First decision 10 July 2017

Revised manuscript received 16 September 2017

Accepted 23 October 2017 